\title{
Editorial
}

\section{Mathematical Modeling and Analysis of Soft Computing}

\author{
Shifei Ding, ${ }^{1,2}$ Zhongzhi Shi, $^{2}$ Ke Chen, ${ }^{3}$ and Ahmad Taher Azar ${ }^{4}$ \\ ${ }^{1}$ School of Computer Science and Technology, China University of Mining and Technology, Xuzhou 221116, China \\ ${ }^{2}$ Key Laboratory of Intelligent Information Processing, Institute of Computing Technology, Chinese Academy of Science, \\ Beijing 100190, China \\ ${ }^{3}$ Centre for Mathematical Imaging Techniques, Department of Mathematical Sciences, University of Liverpool, Liverpool L69 7ZL, UK \\ ${ }^{4}$ Faculty of Computers and Information, Benha University, Benha 13511, Egypt \\ Correspondence should be addressed to Shifei Ding; dingsf@cumt.edu.cn
}

Received 21 December 2014; Accepted 21 December 2014

Copyright (C) 2015 Shifei Ding et al. This is an open access article distributed under the Creative Commons Attribution License, which permits unrestricted use, distribution, and reproduction in any medium, provided the original work is properly cited.

Welcome to this special issue. Soft computing is one of the hot research fields in advanced artificial intelligence, while mathematical modeling and analysis (MMA) plays key role in soft computing. This special issue aims to promote the research, development, and applications of MMA for soft computing by providing a high-level international forum for researchers and practitioners to exchange research results and share development experiences. The papers in this edition were selected from among the highest rated papers in submitted manuscripts. The selection of papers featured here cover the topics of the main soft computing theories and experimental studies of some application systems.

Truss layout optimization is a typical difficult constrained mixed-integer nonlinear program. A. Xiao et al. present fitness estimation based particle swarm optimization algorithm with an adaptive penalty function approach (FEPSOAP). The evaluation of particles is partly substituted by the estimation of similar particles and the iteration information is merged into the penalty function to find a good balance between the exploration and exploitation of the constrained design domain. The present method solves some problems as how we can simplify truss layout optimization and the computational cost of truss analysis. Flower pollination algorithm (FPA) is a new nature-inspired intelligent algorithm which uses the whole update and evaluation strategy on solutions. This algorithm may deteriorate the convergence speed and the quality of solution due to interference phenomena among dimensions for solving multidimension function optimization problems. To overcome this shortage, R. Wang and Y.
Zhou point out a dimension by dimension improvement based flower pollination algorithm.

In view of the small sample classification problem, a neural network ensemble optimized classification algorithm based on partial least squares (PLS) and ordinary least squares (OLS) is proposed by W. Jia et al. This algorithm can reduce the feature dimension of small sample data and improve the recognition precision of classification system. X. Xiang and J. Wu build a framework of moment neuronal networks with intra- and inter-interactions that shows how the spontaneous activity is propagated across the homogeneous and heterogeneous network. The application of chemical flooding in petroleum reservoirs turns into hot topic of the recent researches. In order to improve the estimation efficiency of chemical flooding in oil reservoirs, $M$. A. Ahmadi put forward a predictive model based on swarm intelligence and artificial neural network (ANN).

Multilevel association rules mining is an important domain to discover interesting relations between data elements with multiple levels abstractions. Y. Xu et al. describe novel genetic based multilevel association rules mining to avoid the excessive computation in the big data. The authors use the category tree to describe the multilevel application data sets as the domain knowledge, then put forward a special tree encoding schema based on the category tree to build the heuristic multilevel association mining algorithm, and lastly propose the genetic algorithm based on the tree encoding schema. N. H. Moin et al. propose a hybrid genetic algorithm with multiparents crossover for job shop scheduling problem 
that is one of the well-known hard combinatorial scheduling problems. This algorithm embeds a schedule generation procedure to reduce the search space and ensures the sustainability. Y.-H. Kim and Y. Yoon propose a new distance metric, based on the linkage of genes, in the search space of genetic algorithms. This second-order distance measure is derived from the gene interaction graph and first-order distance, which is a natural distance in chromosomal spaces.

J. Chai et al. design empirical mode decomposition least square support vector machine (EMD LSSVM) model to analyze the CSI 300 index. The EMD-LSSVM model with grid search (GS) algorithm has good performance compared with other optimization methods including simplex, GS, particle swarm optimization (PSO), and genetic algorithm (GA) in predicting stock market movement direction. Focusing on the bifurcation analysis of an SIS epidemic model with bilinear incidence rate and saturated treatment, Y. Xiao et al. point out the global dynamics of an SIS model with bilinear incidence rate and saturated treatment function which shows the effect of delayed treatment when the rate of treatment is lower and the number of infected individuals is getting larger. Z. Shi and Z. Ji point out a new two-stage least squares iterative algorithm to identify the parameters of the identification of Hammerstein finite impulse response moving average (H-FIR-MA) systems. Compared with other methods, the present method can obtain highly accurate parameter estimates and fast convergence rate. Accurate identification of the aggregation residues could help fully decipher the molecular mechanisms. In order to predict aggregation residues from the sequence information, B. Liu et al. introduce two computational approaches: Aggre_Easy and Aggre_Balance. The prediction of aggregation prone sites plays a crucial role in the research of drug-targets.

V. Dobrić et al. present formalization of prototype theory of categorization that is accomplished by using Boolean consistent fuzzy logic-interpolative Boolean algebra. Proposed formalism secures the principles of categorization and shows that Boolean laws are fundamental in the categorization process. H. Wang et al. propose a novel hybrid approach based on mutual information and improved gravitational search algorithm to improve the efficiency and accuracy of CTQs identification. The results of experiment show that the present method has a strong search capability. Accelerated life test is commonly used for the estimation of high-reliability product; the article by G. Wang et al. titled "Accelerated Lifetime Data Analysis with a Nonconstant Shape Parameter" explores a simple and efficient approach to estimate the coefficients of acceleration models by using weight least square method.

The traditional social cognitive optimization (SCO) is not guaranteed to converge to the global optimization solution with probability one. J. Sun et al. design an improved SCO algorithm which is guaranteed to converge to the global optimization solution and has better stable optimization outcomes than the traditional SCO for nonlinear programming problems. The convergence proof for the improved SCO based on Solis and Wets' research results is given. F.-A. Deng et al. propose an improved harmony search algorithm based on NGHS algorithm (INGHS). The novel harmony search algorithm has a good performance for optimization problems into which nine construction examples of algebra system are converted. Global best strategy and dynamic parameters adjustment are employed in INGHS.

Many scholars have made significant contributions to research, education, and development of soft computing. This special issue is dedicated to those pioneers and scientific and technical workers in research in this area.

\section{Acknowledgments}

This special issue is supported by the National Natural Science Foundation of China under Grant no. 61379101 and the National Basic Research Program of China under Grant no. 2013CB329502. Finally, we would like to thank all those who helped to make this special issue possible, especially the authors and the reviewers of the articles.

Shifei Ding

Zhongzhi Shi

Ke Chen

Ahmad Taher Azar 


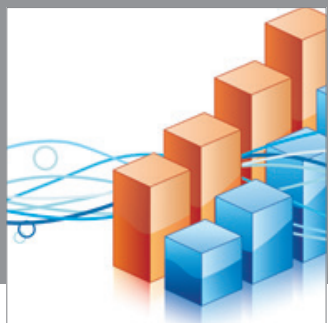

Advances in

Operations Research

mansans

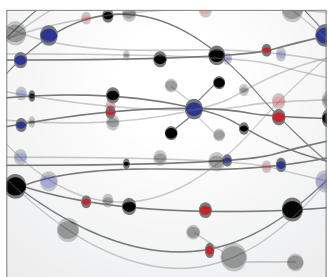

The Scientific World Journal
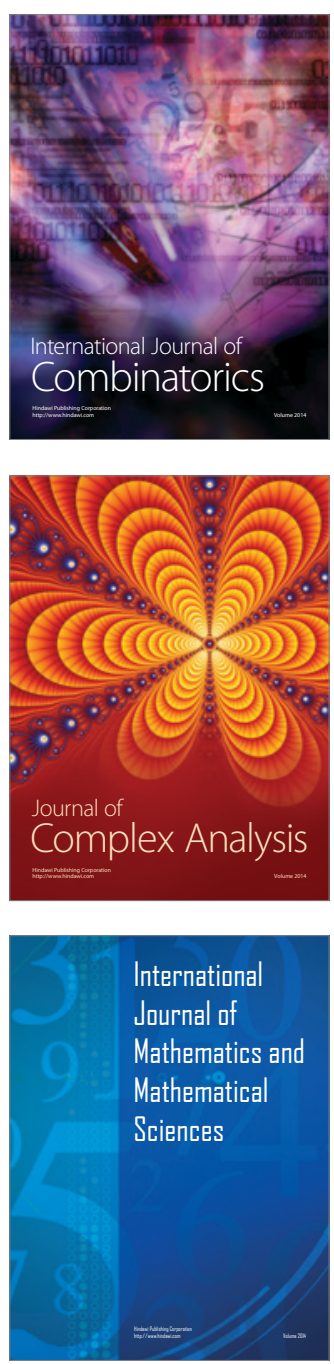
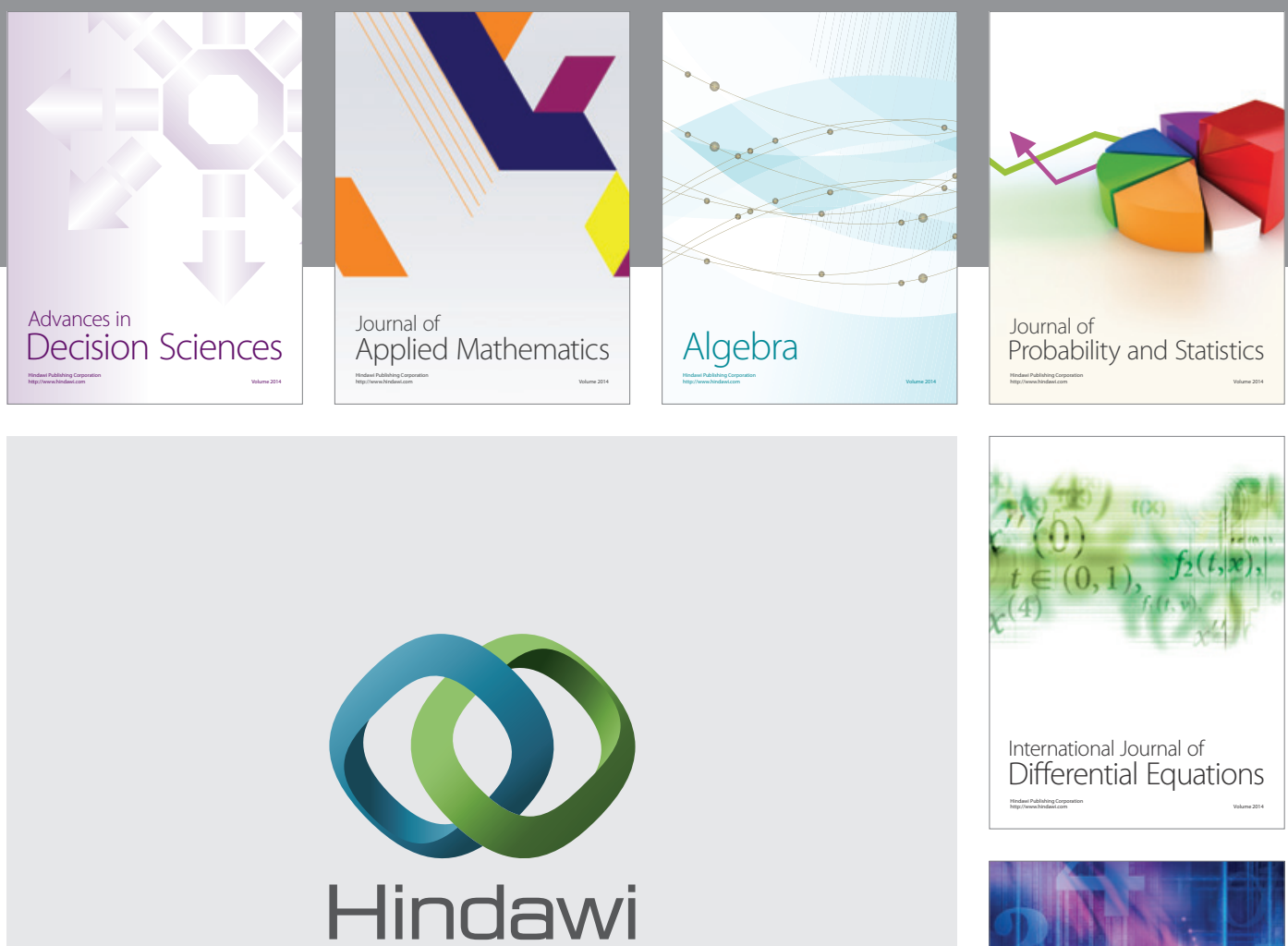

Submit your manuscripts at http://www.hindawi.com
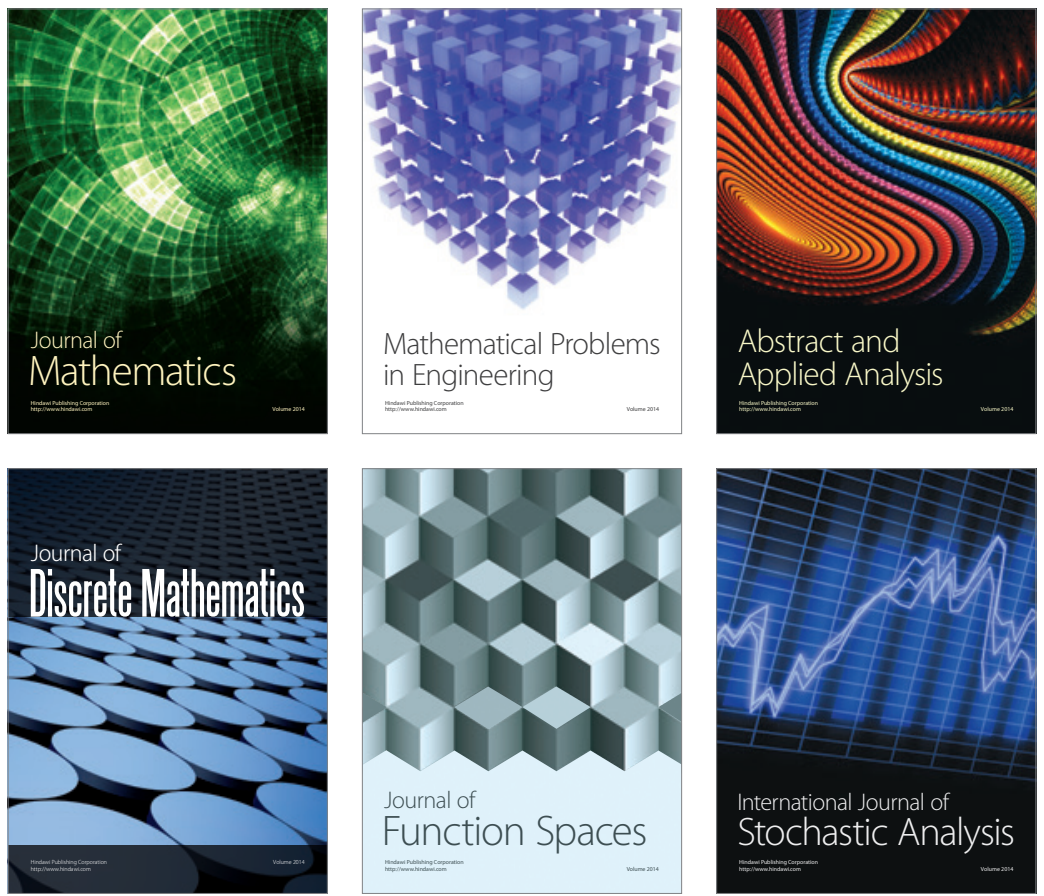

Journal of

Function Spaces

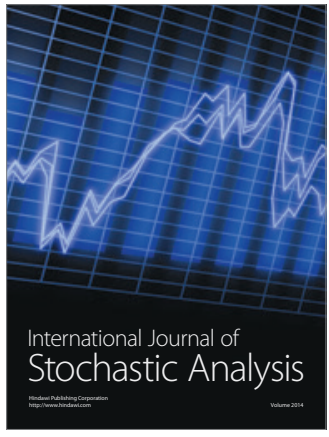

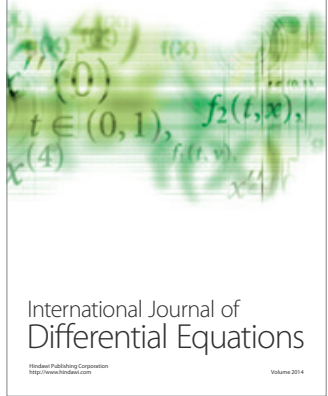
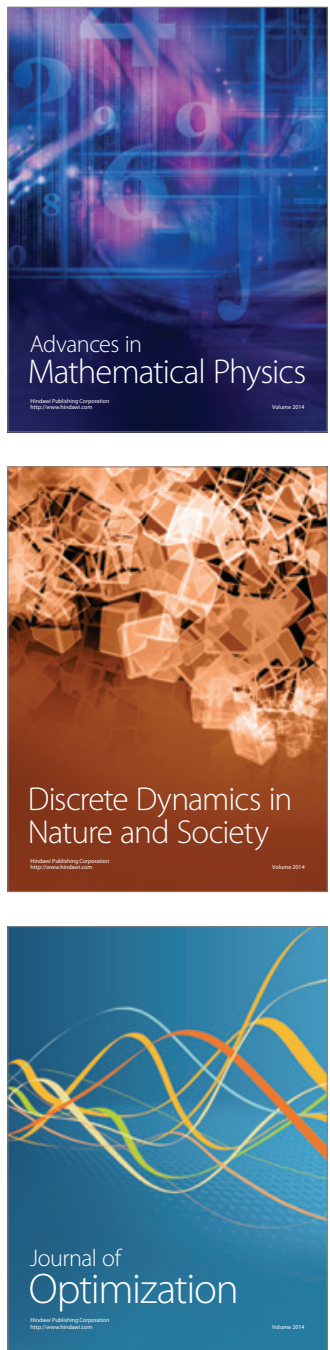